\title{
Second-order Born approximation for the ionization of molecules by electron and positron impact
}

\author{
C. Dal Cappello, ${ }^{1}$ Z. Rezkallah, ${ }^{2}$ S. Houamer, ${ }^{2,}{ }^{*}$ I. Charpentier, ${ }^{3}$ P. A. Hervieux, ${ }^{4}$ M. F. Ruiz-Lopez, ${ }^{5}$ \\ R. Dey, ${ }^{6}$ and A. C. Roy ${ }^{7}$ \\ ${ }^{1}$ Université Paul Verlaine-Metz, Laboratoire de Physique Moléculaire et des Collisions, \\ Institut Jean Barriol (FR2843), 1 Boulevard Arago, F-57078 Metz Cedex 3, France \\ ${ }^{2}$ Laboratoire de Physique Quantique et Systèmes Dynamiques, Département de Physique, Faculté \\ des Sciences Université Ferhat Abbas, Sétif 19000, Algeria \\ ${ }^{3}$ Université Paul Verlaine-Metz, Laboratoire de Physique et Mécanique des Matériaux UMR 7554, \\ Ile du Saulcy, F-57045 Metz Cedex 1, France \\ ${ }^{4}$ Institut de Physique et Chimie des Matériaux de Strasbourg, 23 Rue du Loess, BP 43, F-67034 Strasbourg Cedex 2, France \\ ${ }^{5}$ Nancy-University, Equipe de Chimie et Biochimie Théoriques, UMR CNRS-UHP 7565, BP 239, \\ F-54506 Vandoeuvre-les-Nancy, France \\ ${ }^{6}$ Max-Planck Institut für Plasmaphysik, Boltzmannstr. 2, D-85748 Garching, Germany \\ ${ }^{7}$ School of Mathematical Sciences, Ramakrishna Mission Vivekananda University, Belur Math 711202, West Bengal, India
}

(Received 26 June 2011; published 20 September 2011)

\begin{abstract}
Second-order Born approximation is applied to study the ionization of molecules. The initial and final states are described by single-center wave functions. For the initial state a Gaussian wave function is used while for the ejected electron it is a distorted wave. Results of the present model are compared with recent $(e, 2 e)$ experiments on the water molecule. Preliminary results are also presented for the ionization of the thymine molecule by electrons and positrons.
\end{abstract}

DOI: 10.1103/PhysRevA.84.032711

PACS number(s): $34.80 . \mathrm{Dp}$

\section{INTRODUCTION}

Ionization of molecules by light charged particles (electrons and positrons) is a difficult problem and is a true challenge for theoreticians. One of the difficulties is that the molecular states are described by multicenter wave functions. A way to avoid this difficulty is to replace the multicenter wave function by a single-center one. For instance, Hafied et al. [1] and Dal Cappello et al. [2] have been able to overcome this difficulty in their calculations for the water molecule and for the cytosine molecule, respectively. In the first case it was easy because the center of the water molecule was considered to lie at the center of the oxygen atom, and a good convergence was reached using only two partial waves (i.e., $L=0$ and $L=1$ ). In the second case the convergence was reached with an increasing number of partial waves (i.e., with $L=0$ to $L=5$ ) in their study of the ionization of cytosine by protons.

In this paper we extend our first Born model to the next higher second Born model, because the energy of incident electrons used in the recent $(e, 2 e)$ experiments of MilneBrownlie et al. [3], Kaiser et al. [4], and Nixon et al. [5] for the ionization of the water molecule is small (from $30 \mathrm{eV}$ to $250 \mathrm{eV}$ ). For this energy range one needs the use of theories more appropriate than the first Born approximation. For instance, the lack of symmetry about the momentum transfer in the experimental data demands that the second born approximation is at least necessary. Experiments on the ionization of thymine by electron impact are in progress in the laboratory of Lohmann (see for instance [6]) and are planned to be performed at an incident energy of $250 \mathrm{eV}$. In this kind of $(e, 2 e)$ experiment the ejected electron is detected in coincidence with the scattered electron. For the

*Corresponding author: hosalim@yahoo.com above kinematical arrangement the ejected electrons have low velocities (their energies vary between $10 \mathrm{eV}$ and $20 \mathrm{eV}$ ) contrary to those of the scattered electrons (their energies are close to $200 \mathrm{eV}$ ). This allows us to ignore the exchange effects.

At this time, there is no quantum mechanical theory for the ionization of thymine or other DNA bases. Nevertheless total cross sections for the four bases of DNA (adenine, cytosine, guanine, and thymine) have been calculated by using semiclassical models. Bernhardt and Paretzke [7] have used the semiclassical Deutsch-Märk (DM) formalism [8] and the binary-encounter-Bethe (BEB) theory [9]. These simple models can be applied to determine the electron impact ionization cross sections for various atoms, molecules, and ions. They only need molecular structure information, which can be provided by the Hartree-Fock method. However, we notice that the BEB and the DM models are not quite successful in describing the last experiments of Shafranyosh et al. [10] for the ionization of the cytosine molecule by electrons. These two models yield a maximum total cross section of $15 \times 10^{-16}$ $\mathrm{cm}^{2}$ against $8 \times 10^{-16} \mathrm{~cm}^{2}$ found in the experiments. Even the more recently improved binary-encounter dipole (IBED) model [11] gives a maximum total cross section of $17 \times 10^{-16}$ $\mathrm{cm}^{2}$ [12] although this model perfectly reproduces the total cross section of the water molecule [12]. The IBED model differs from the BEB model in two main aspects. First, the IBED treatment takes into account the long-range electrontarget dipole interaction (as in the BEB model) in addition to the shielding of the dipole field as the scattering electron comes inside the bonding region. Secondly, this model predicts an $\left(E_{e}\right)^{-3.5}$ ejected electron energy dependence instead of $\left(E_{e}\right)^{-3}$ as in the BEB model for the optical oscillator strength.

It is worth stressing that all these models (DM, BEB, and IBED) are unable to calculate triple differential cross sections (TDCSs) which are measured in $(e, 2 e)$ experiments. 
To date the most accurate model to calculate the TDCS is the molecular three-body distorted-wave approach (M3DW) $[13,14]$, which is a generalization of the three-body distortedwave approximation (3DW) [15] for molecules. In the 3DW any interaction included in the calculation of both the initial state and the final state is contained to all orders of perturbation theory while all interactions contained in the perturbation operator are of first order in perturbation theory. As the TDCS depends on molecular orientation, the usual $(e, 2 e)$ measurements provide an average over all molecular orientations. However, the M3DW model needs a lot of computer time and Gao et al. [13] proposed the orientation-averaged molecular orbital (OAMO) where a single average molecular orbital is used to approximate the average over all orientations. This approximation is only successful for a few highly symmetric states. For instance, this approximation is not valid for the $1 b_{1}$ state of the water molecule [14]. For the case of the ionization of the water molecule other less sophisticated models were applied. Champion et al. [16] used the distorted-wave Born approximation (DWBA) where the incident and scattered electrons are described by plane waves while the ejected electron is described by a distorted wave. But this model was unable to reproduce the recoil peak in the experiments of Milne-Brownlie et al. [3] because Champion et al. [16] neglected the interaction between the incident electron and the nucleus of the target. When this last interaction was included in the DWBA model a better agreement was found [17]. Champion et al. [17] also introduced the well-known BraunerBriggs-Klar (BBK) model [18] where all the interactions have been taken into account: the interaction of the ionized target with the projectile electron as well as the ejected electron and the repulsion between the outgoing electrons. In this BBK model the scattered electron and the ejected electron are described by a Coulomb wave while in the M3DW model distorted waves are used.

In this paper we decided to use the second Born approximation, which is more tractable than the BBK model for complex molecules such as DNA bases. The ionization of such a molecule is important for life science because it is now known [19] that low-energy secondary electrons (less than $20 \mathrm{eV}$ ) strongly interact with biological molecules in the DNA via dissociative electron attachment [20]. These interactions lead to single or double DNA strand breakage.

In Sec. II we present our theoretical model to describe the ionization of a molecule by electrons or positrons. Then, in Sec. III, the TDCS results are compared with experimental data for the ionization of water. We also present preliminary results for the ionization of thymine. Finally, conclusions about the modelling of the ionization of molecules by electrons and positrons are outlined in Sec. IV.

Atomic units are used throughout unless otherwise indicated.

\section{THEORY}

The ionization of a molecule $M$ by an electron (or a positron) can be considered as a pure electronic transition since the closure relation over all possible rotational and vibrational states of the residual target can be applied. This is justified by the values of the collision time compared to the characteristic time of rotation and vibration. The exchange effects will be neglected since the scattered electron is much faster than the ejected one in all the cases considered here.

The single ionization of a molecule $M$ by an electron is written as

$$
M+e^{-} \rightarrow M^{+}+e^{-}+e^{-} .
$$

In the second Born approximation (SBA), the four-fold differential cross section (4DCS) is written, for a given molecular orientation defined by the Euler angles $(\alpha ; \beta ; \gamma)$, as

$$
\begin{aligned}
\sigma^{(4)}(\alpha ; \beta ; \gamma) & =\frac{d^{4} \sigma(\alpha ; \beta ; \gamma)}{d \Omega_{\text {Euler }} d \Omega_{s} d \Omega_{e} d E_{e}} \\
& =\frac{k_{s} k_{e}}{k_{i}}\left|f_{B 1}+f_{B 2}\right|^{2},
\end{aligned}
$$

where $d \Omega_{\text {Euler }}=\sin \beta d \beta d \alpha d \gamma$ and $d \Omega_{s}$ and $d \Omega_{e}$ denote the elements of solid angles for the scattered and the ejected electron, respectively, whereas the energy interval of the ejected electron is represented by $d E_{e}$. The momenta of the incident, the scattered, and the ejected electrons are denoted by $\vec{k}_{i}, \vec{k}_{s}$, and $\vec{k}_{e}$, respectively.

In an $(e, 2 e)$ reaction the conservation of energy imposes $\frac{k_{i}^{2}}{2}=\frac{k_{s}^{2}}{2}+\frac{k_{e}^{2}}{2}+I_{i}$ where $I_{i}$ represents the energy needed to eject one electron from the molecule.

The first Born term $f_{B 1}$ is written as

$$
\begin{aligned}
f_{B 1}= & -\frac{1}{2 \pi}\left\langle\exp \left(i \vec{k}_{s} \cdot \vec{r}_{0}\right) \Psi_{f}\left(\vec{k}_{e}, \vec{r}_{1}, \ldots, \vec{r}_{n}\right)\right| \\
& \times V\left|\exp \left(i \vec{k}_{i} \cdot \vec{r}_{0}\right) \Phi_{i}\left(\vec{r}_{1}, \ldots, \vec{r}_{n}\right)\right\rangle,
\end{aligned}
$$

where $\Phi_{i}\left(\vec{r}_{1}, \ldots, \vec{r}_{n}\right)$ is the wave function of the initial state of the molecule while $\Psi_{f}\left(\vec{k}_{e}, \vec{r}_{1}, \ldots, \vec{r}_{n}\right)$ represents the wave function for the single continuum state of the molecule.

In Eq. (3), the potential $V$ represents the Coulomb interaction between the incoming electron and the target and is written as

$$
V=-\frac{Z}{r_{0}}-\sum_{j=1}^{N} \frac{1}{\left|\vec{r}_{0}-\vec{R}_{j}\right|}+\sum_{i=1}^{n} \frac{1}{\left|\vec{r}_{0}-\vec{r}_{i}\right|},
$$

where $\vec{r}_{i}$ is the position vector of the $i$ th bound electron of the target with respect to the centre of the molecule, $\vec{r}_{0}$ denotes the coordinate of the incident particle and $\vec{R}_{j}$ is the position of the $j$ th nucleus.

The second Born term $f_{B 2}$ is given by

$$
\begin{aligned}
f_{B 2}= & \frac{1}{8 \pi^{4}} \sum_{m} \int \frac{d \vec{q}}{q^{2}-k_{m}^{2}-i \varepsilon} \\
& \times\left\langle\exp \left(i \vec{k}_{s} \cdot \vec{r}_{0}\right) \Psi_{f}\left(\vec{k}_{e}, \vec{r}_{1}, \ldots, \vec{r}_{n}\right)\right| \\
& \times V\left|\exp \left(i \vec{q} \cdot \vec{r}_{0}\right) \Phi_{m}\left(\vec{r}_{1}, \ldots, \vec{r}_{n}\right)\right\rangle \\
& \times\left\langle\exp \left(i \vec{q} \cdot \vec{r}_{0}\right) \Phi_{m}\left(\vec{r}_{1}, \ldots, \vec{r}_{n}\right)\right| \\
& \times V\left|\exp \left(i \vec{k}_{i} \cdot \vec{r}_{0}\right) \Phi_{i}\left(\vec{r}_{1}, \ldots, \vec{r}_{n}\right)\right\rangle,
\end{aligned}
$$

where the summation over $m$ means that we take into account all the contributions of the $m$ discrete and continuum states of the molecule, $\Phi_{m}\left(\vec{r}_{1}, \ldots, \vec{r}_{n}\right)$ being the wave function for a discrete or continuum state of the molecule. It means that the incident electron collides two times with the target. 
The reduction of this difficult $n$-electron target problem to a single electron target may be done within the well-known frozen-core approximation. In this case this target electron will be ejected during the single ionization process. Moreover, it is worthwhile to note that the electrons in the singly charged ion core are assumed to remain unaffected by the ionization process. This is a reasonable approximation provided that our study is limited to the ejection of valence shell electrons.

Note that the integration over the projectile coordinates $\left(r_{0}\right)$ can be performed analytically so that the second Born term, by applying the closure approximation [21], becomes

$$
\begin{aligned}
\vec{f}_{B 2}= & \frac{2}{\pi^{2}} \int \frac{d \vec{q}}{q^{2}-p^{2}-i \varepsilon} \frac{1}{K_{i}^{2} K_{f}^{2}} \\
& \times\left\langle\Psi_{C}^{-}\left(\vec{k}_{e}, \vec{r}_{1}\right)\right| \exp \left(i \vec{K}_{f} \cdot \vec{r}_{1}\right)-1 \\
& \left.\times\left|\exp \left(i \vec{K}_{i} \cdot \vec{r}_{1}\right)-1\right| \Phi_{i}\left(\vec{r}_{1}\right)\right\rangle
\end{aligned}
$$

where $\vec{K}_{i}=\vec{k}_{i}-\vec{q}$ and $\vec{K}_{f}=\vec{q}-\vec{k}_{s}$, and $\vec{K}=\vec{K}_{i}+$ $\vec{K}_{f}=\vec{k}_{i}-\vec{k}_{s}$ is the momentum transfer.

We have also

$$
\frac{p^{2}}{2}=\frac{k_{i}^{2}}{2}-\bar{w}
$$

where $\bar{w}$ refers to the average excitation energy. In our study we have $\bar{w}=I_{i}$ which corresponds to the usual choice for the single ionization of an atom or a molecule [28].

When we consider a single electron target it is also possible to apply the eikonal approximation (EA) [22]. This approximation is valid for small momentum transfer to the target and sufficiently high incident energy [23]. The 4DCS in the eikonal approximation [23] is given by

$$
\sigma^{(4)}(\alpha ; \beta ; \gamma)=\frac{d^{4} \sigma(\alpha ; \beta ; \gamma)}{d \Omega_{\mathrm{Euler}} d \Omega_{s} d \Omega_{e} d E_{e}}=\frac{k_{s} k_{e}}{k_{i}}\left|T_{f i}\right|^{2},
$$

where

$$
\begin{aligned}
T_{f i}= & -\frac{1}{2 \pi}\left\langle\exp \left(i \vec{k}_{s} \cdot \vec{r}_{0}\right) \Psi_{C}^{-}\left(\vec{k}_{e}, \vec{r}_{1}\right)\right. \\
& \times\left\lfloor\frac{1}{r_{01}}-\frac{1}{r_{0}}\left|\exp \left(i \vec{k}_{i} \cdot \vec{r}_{0}\right) \Phi_{i}\left(\vec{r}_{1}\right)\right\rangle\right. \\
& \times\left\lfloor\frac{r_{01}-\vec{r}_{01} \cdot \hat{z}}{r_{1}-\vec{r}_{1} \cdot \hat{z}}\right]^{i / k_{i}},
\end{aligned}
$$

with $\hat{z}=\frac{\vec{k}_{i}}{k_{i}}$. In contrast to the first Born approximation (FBA), the EA, which has been applied with success to a wide variety of atomic collisions including elastic scattering, excitation, and ionization has contributions from projectile-target nucleus interaction and multiple scattering effects. In fact, the eikonal amplitude contains terms of all orders in $V$ (i.e., the sum of the projectile-nucleus interaction and the projectile-bound electron interaction) in its phase in an approximate way.

The water and thymine wave functions have been obtained using the GAUSSIAN 03 program [24] as follows. In the case of water, the wave function has been computed at the Hartree-Fock level of theory using the augmented, correlationconsistent, polarized-valence quadruple-zeta basis set (augcc-pvQZ) employed in our previous work [1]. Geometry optimization has been done including electronic correlation energy at the second-order Møller-Plesset perturbation theory (MP2). For thymine, we have chosen a lower computational level that we previously used for cytosine [2]. The geometry of the molecule has also been optimized at the MP2 level but using now the 6-31G(d) basis set that includes a doublezeta valence shell and polarization orbitals on nonhydrogen atoms. The molecular orbitals hereafter correspond to the Hartree-Fock calculations. The multicenter wave function is then converted to a single-center expansion (see Appendix) of usual Slater-type functions by using partial-wave expansion techniques $[1,25]$. Here we restrict this study to the valence electrons because inner shell electrons need many partial waves for the expansion.

All the one-center molecular wave functions $\Phi_{i}(\vec{r})$ (with $i$ ranging from 1 to 48 for thymine), containing the valence electrons, can be expressed by linear combinations of Slatertype functions and are written as

$$
\Phi_{i}(\vec{r})=\sum_{k=1}^{N_{i}} a_{i k} \phi_{n_{i k} \varepsilon_{i k} m_{i k}}^{\varepsilon_{i k}}(\vec{r}),
$$

where $N_{i}$ is the number of Slater functions used in the construction of the $j$ th molecular orbital and $a_{i k}$ the weight of each complex atomic component $\varphi_{n_{i k} l_{i k} m_{i k}}^{\varepsilon_{i k}}(\vec{r})$.

In Eq. (10), $\varphi_{n_{i k} l_{i k} m_{i k}}^{\varepsilon_{i k}}(\vec{r})$ is written as

$$
\phi_{n_{i k} l_{i k} m_{i k}}^{\varepsilon_{i k}}(\vec{r})=\left[R_{n_{i k}}^{\xi_{i k}}(r)+i S_{n_{i k}}^{\xi_{i k}}(r)\right] Y_{l_{i k}}^{m_{i k}}(\hat{r}),
$$

where the radial part $\left[R_{n_{i k}}^{\xi_{i k}}(r)+i S_{n_{i k}}^{\xi_{i k}}(r)\right]$ can be given by the usual radial Slater-type functions such as $b_{n_{i k}} r^{n_{i k}-1} \exp \left(-\varepsilon_{i k} r\right)$. We notice that here the generated wave function is generally complex whereas it was real in the case of Moccia' wave functions [26].

In our present model the scattered electron is described by a plane wave, whereas the ejected electron can be described by a distorted wave or a Coulomb wave. Owing to the prohibitively long time needed for the calculation of the second Born term we only use a Coulomb wave for the ejected electron

$$
\begin{aligned}
\phi_{C}\left(\vec{k}_{e}, \vec{r}_{1}\right)= & \frac{\exp \left(i \vec{k}_{e} \cdot \vec{r}_{1}\right)}{(2 \pi)^{3 / 2}}{ }_{1} F_{1} \\
& \times\left[-i Z_{e} / k_{e}, 1,-i\left(\vec{k}_{e} \cdot \vec{r}_{1}+k_{e} r_{1}\right)\right] \\
& \times \exp \left(\frac{\pi Z_{e}}{2 k_{e}}\right) \Gamma\left(1+i Z_{e} / k_{e}\right) .
\end{aligned}
$$

The effective ionic charge $Z_{e}$ is taken to be equal to 1 (Brothers and Bonham [27]).

For the positron impact the only change in our model is to write $V$ as

$$
V=\frac{Z}{r_{0}}+\sum_{j=1}^{N} \frac{1}{\left|\vec{r}_{0}-\vec{R}_{j}\right|}-\sum_{i=1}^{n} \frac{1}{\left|\vec{r}_{0}-\vec{r}_{i}\right|},
$$

Under these conditions, it is clear that exactly the same 4DCS will be obtained for electrons and positrons when the single ionization process is described within the first Born approximation. On the contrary, when the second term of Born series is used, differences will appear between electron and positron 4DCSs since the sign of the second Born term does not depend on the charge of the particle. 
Finally, it is important to note that in the second Born term the integrals over $d \vec{q}$ must be performed numerically with great care [28] since the integrand is singular at $q=k_{m}$ [Eq. (5)] or $q=p[\mathrm{Eq}$. (6)].

The wave functions $\Phi_{i}(\vec{r})$ correspond to a particular orientation of the molecular target given by the Euler angles $(\alpha$, $\beta, \gamma)[29,30]$. Thus, the four differential cross sections we have calculated with Eqs. (2)-(10) correspond to the ionization of an oriented molecule. Under these conditions, we need to average these differential cross sections in order to compare with experiment. The averaging is accomplished by an analytical integration over the Euler angles, owing to the property of the rotation matrix [17,29,30].

$$
\begin{aligned}
& \frac{1}{8 \pi^{2}} \int d \Omega D_{\mu, m}^{l}(\alpha, \beta, \gamma) D_{\mu^{\prime}, m^{\prime}}^{l^{\prime *}}(\alpha, \beta, \gamma) \\
& \quad=\frac{1}{2 l+1} \delta_{l, l^{\prime}} \delta_{m, m^{\prime}} \delta_{\mu, \mu^{\prime}},
\end{aligned}
$$

where $D_{\mu, m}^{l}(\alpha, \beta, \gamma)$ represents the rotation matrix with $Y_{m}^{l}(\hat{r})=\sum_{\mu=-l}^{l} D_{\mu, m}^{l}(\alpha, \beta, \gamma) Y_{\mu}^{l}(\hat{r})$ for the transformation of the molecular orientation from the molecular frame to the laboratory frame. Equation (14) can also be applied to our models including the second Born approximation with the closure approximation [Eq. (6)] as for other sophisticated models as the continuum distorted wave (CDW) eikonal initial state (EIS) approximation [31,32] or BBK [17]. These two models (CDW-EIS and BBK) need a six-dimensional integral. In general, the DWBA model gives reasonable agreement with the TDCS measurements for electron-impact ionization of atoms and molecules if the incident electron has an energy of about $100 \mathrm{eV}$ or larger [14].

\section{RESULTS AND DISCUSSION}

Our model is first applied to the ionization of the water molecule. Recently $(e, 2 e)$ experiments were performed by Milne-Brownlie et al. [3] for the $2 a_{1}$ state, the $1 b_{1}$ state, the $1 b_{2}$ state and the summed $3 a_{1}+1 b_{1}$ states for $250 \mathrm{eV}$ incident particles and slow ejected electrons ( $8 \mathrm{eV}$ and $10 \mathrm{eV})$. Kaiser et al. [4] reported results only for the $1 b_{1}$ state at incident electron energies varying between $30 \mathrm{eV}$ and $110 \mathrm{eV}$. Nixon et al. [5] were able to measure TDCSs for the $3 a_{1}$ state at low energies (from $4 \mathrm{eV}$ to $40 \mathrm{eV}$ above threshold). We restrict our present study to the higher incident energy $(250 \mathrm{eV})$ where several models have been applied. The DWBA model of Champion et al. $[3,16]$ was able to reproduce the binary peak but not the recoil structure experimentally observed. When the interaction between the incident electron and the target nucleus was taken into account the agreement was better [17] but some discrepancies still remained. Finally, when the BBK $[17,18]$ and the DS3C model (BBK with effective charges) [17,33,34] were applied, the agreement improved particularly for the ionization of the $2 a_{1}$ state. These models were applied using the one-center wave function of Moccia [26]. Our aim is to show that the present models (eikonal approximation and second Born approximation) are also able to predict TDCSs in good agreement with experiment. All the TDCSs are given in atomic units.

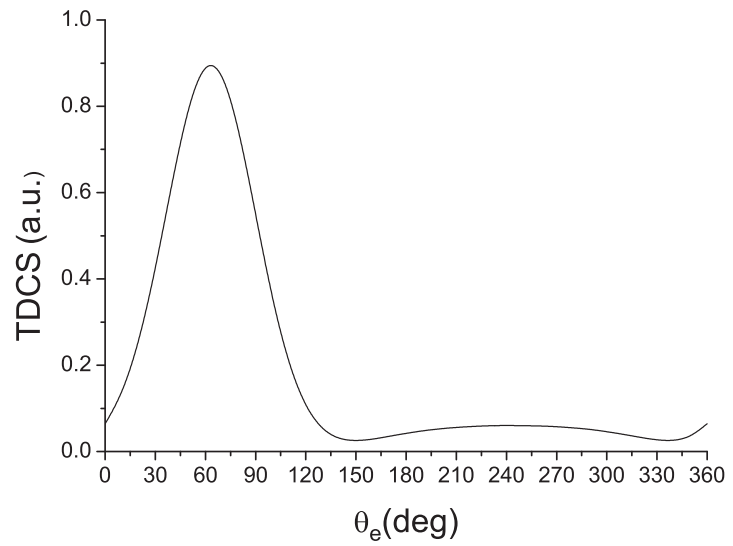

(a)

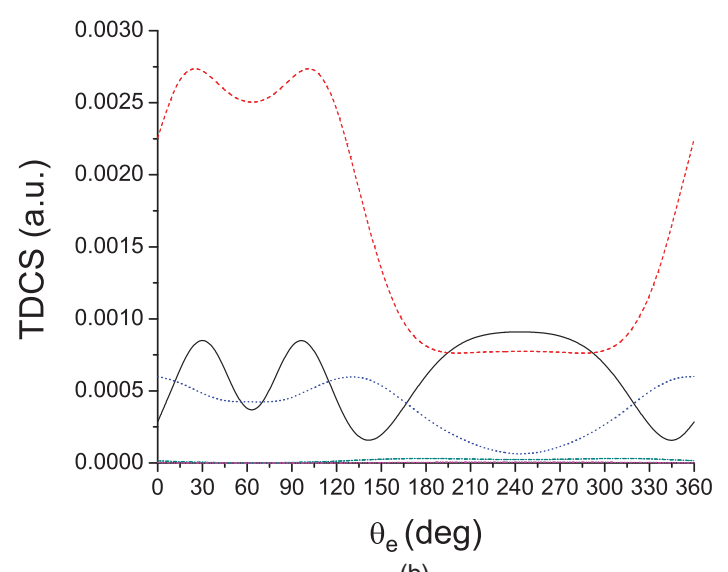

(b)

FIG. 1. (Color online) (a) Triple differential cross section for $250 \mathrm{eV}$ electron-impact ionization of the $2 a_{1}$ orbital of the water molecule. The theoretical calculation is performed in the first Born approximation ( $L=0$ partial-wave contribution). The energy of the ejected electron is $E_{e}=10 \mathrm{eV}$ and the scattered angle is $15^{\circ}$. (b) Triple differential cross section for $250 \mathrm{eV}$ electron-impact ionization of the $2 a_{1}$ orbital of the water molecule. The theoretical calculation is performed in the first Born approximation. The energy of the ejected electron is $E_{e}=10 \mathrm{eV}$ and the scattered angle is $15^{\circ}$. Contribution of $L=1$ (solid line), of $L=2$ (dashed line), of $L=3$ (dotted line), and of $L=4$ (dashed-dotted line).

Figures 1(a) and 1(b) show that the partial wave expansion of the multicenter wave function corresponding to the initial state $2 a_{1}$ is simply reduced to $L=0$, the other terms $(L=1$ to $L=5$ ) being negligible. It is interesting to notice that the wave function of Moccia for the initial state $2 a_{1}$ is mainly built of $L=0$ too. Figure 2(a) shows the results of the first and second Born approximations together with those of the eikonal approximation. The shift of the binary peak is well reproduced by our second Born approximation. The eikonal approximation without the post collisional interaction (PCI) is not able to reproduce such a shift. Compared to the BBK or DS3C models, our models including the second Born approximation or the eikonal approximation slightly underestimate the magnitude of the recoil peak. Figure 2(b) presents an interesting comparison between the TDCS obtained for the electron impact and for the positron impact. As in the case of the ionization of the atomic hydrogen $[18,28]$ we find that the magnitude of the 


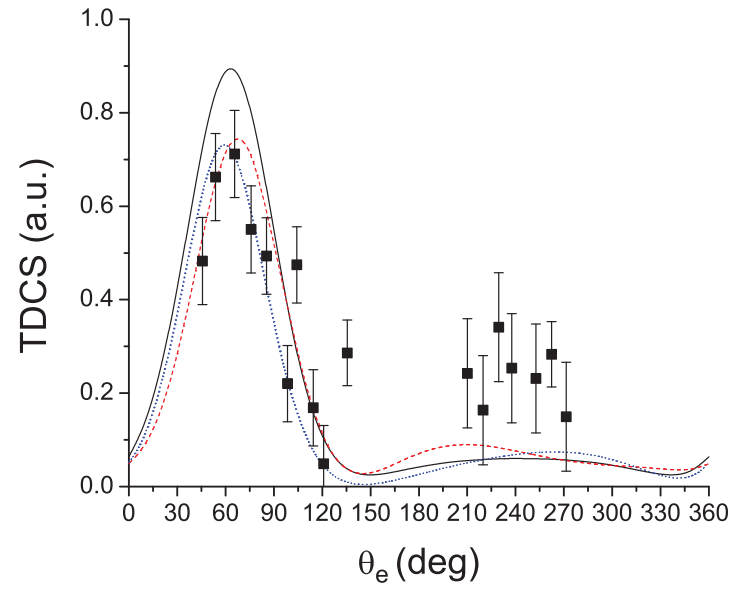

(a)

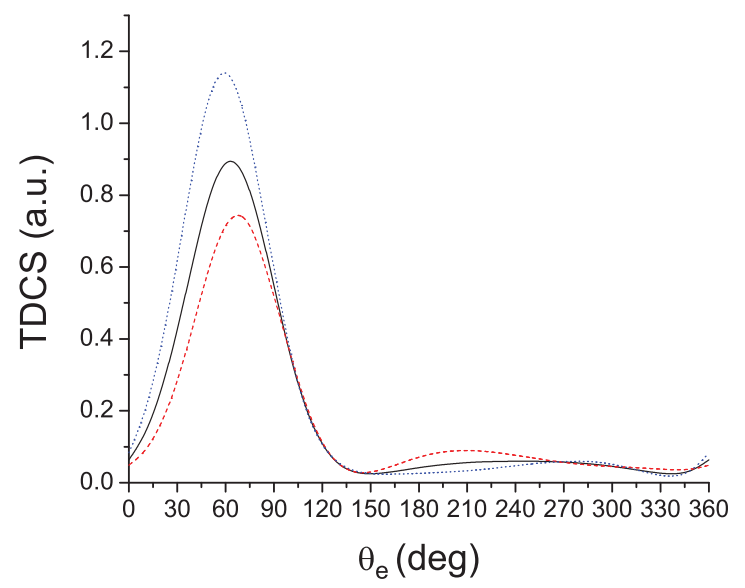

(b)

FIG. 2. (Color online) (a) Triple differential cross section for $250 \mathrm{eV}$ electron-impact ionization of the $2 a_{1}$ orbital of the water molecule. The energy of the ejected electron is $E_{e}=10 \mathrm{eV}$ and the scattered angle is $15^{\circ}$. The theoretical calculations are performed in the first Born approximation (solid line), in the second Born approximation (dashed line), and with the eikonal approximation (dotted line). The experimental data are those of Milne-Brownlie et al. [3]. (b) Triple differential cross section for $250 \mathrm{eV}$ electronimpact and positron-impact ionization of the $2 a_{1}$ orbital of the water molecule. The energy of the ejected electron is $E_{e}=10 \mathrm{eV}$ and the scattered angle is $15^{\circ}$. The theoretical calculations are performed in the first Born approximation (solid line) and in the second Born approximation for electron impact (dashed line) and for positron impact (dotted line).

binary peak increases for the positron impact while that of the recoil peak decreases.

Figure 3(a) shows the summed TDCS of our second Born approximation for the ionization of the sum of the two states $3 a_{1}$ and $1 b_{1}$. We find good agreement between our model and the data of Milne-Brownlie et al. [3]. We notice that the symmetry around the momentum transfer is destroyed and that a double lobe of the binary peak appears. The magnitude of the second peak increases while that for the first peak decreases. This is admittedly a typical characteristic feature of the second Born approximation for the $p$ orbital. In the present case, both the $3 a_{1}$ and the $1 b_{1}$ are mainly dealt with the $L=1$ partial-wave expansion. When we consider the

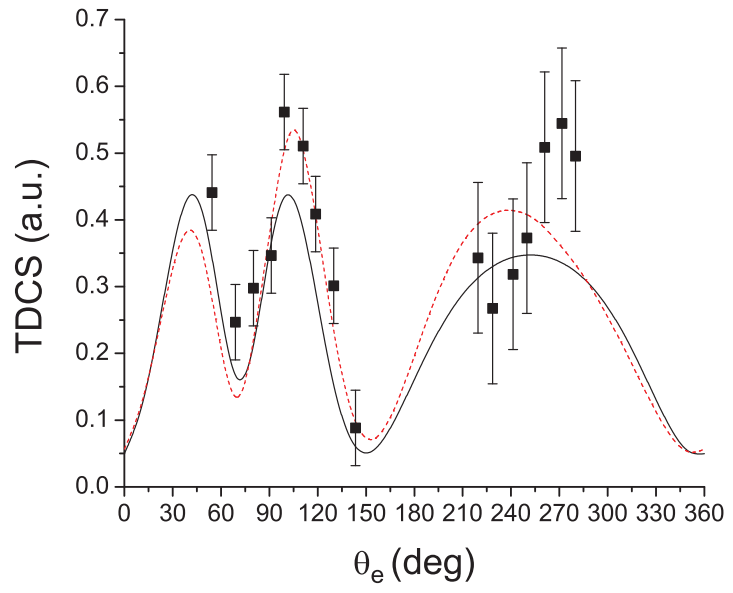

(a)

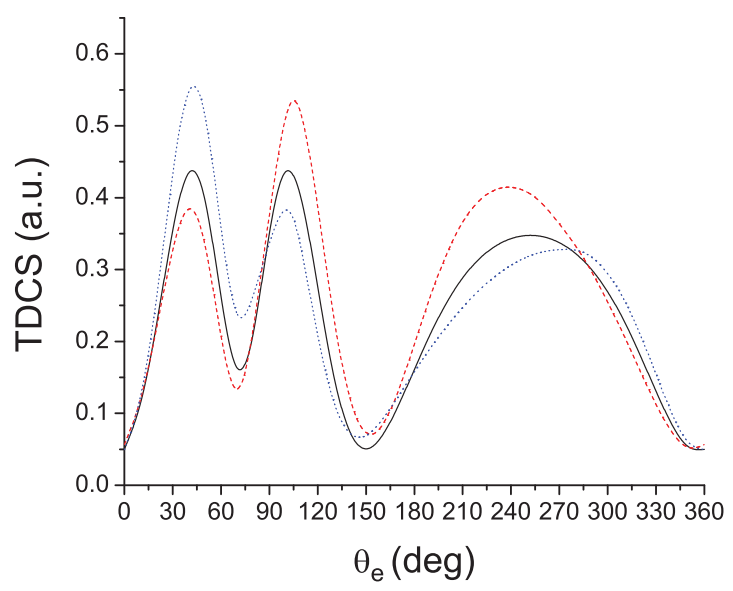

(b)

FIG. 3. (Color online) (a) Summed triple differential cross section for $250 \mathrm{eV}$ electron-impact ionization of the $1 b_{1}$ and $3 a_{1}$ orbitals of the water molecule. The energy of the ejected electron is respectively $E_{e}=10 \mathrm{eV}$ for the $1 b_{1}$ orbital and $8 \mathrm{eV}$ for the $3 a_{1}$ orbital. The scattered angle is $15^{\circ}$. The theoretical calculations are performed in the first Born approximation (solid line) and in the second Born approximation (dashed line). The experimental data are those of Milne-Brownlie et al. [3]. (b) Summed triple differential cross section for $250 \mathrm{eV}$ electron-impact ionization of the $1 b_{1}$ and $3 a_{1}$ orbitals of the water molecule. The energy of the ejected electron is respectively $E_{e}=10 \mathrm{eV}$ for the $1 b_{1}$ orbital and $8 \mathrm{eV}$ for the $3 a_{1}$ orbital. The scattered angle is $15^{\circ}$. The theoretical calculations are performed in the first Born approximation (solid line) and in the second Born approximation for electron impact (dashed line) and for positron impact (dotted line).

ionization of the two states $3 a_{1}+1 b_{1}$ by positrons [Fig. 3(b)] we see a complete change in the structure of the binary peak: The first peak increases while the second peak decreases. We notice that the recoil peak decreases too. Due to many parametric differentiations [35] the eikonal approximation is not applied for the ionization of the $1 b_{1}, 1 b_{2}$, and $3 a_{1}$ states.

Figure 4(a) for the ionization of the $1 b_{1}$ state by electrons gives the same pattern as in Fig. 3(a). We nevertheless see that the agreement with experiment is not quite as good in the small-ejected angle region. Figure 4(b) displays a comparison of the TDCSs for the $1 b_{1}$ state obtained in the FBA and SBA 


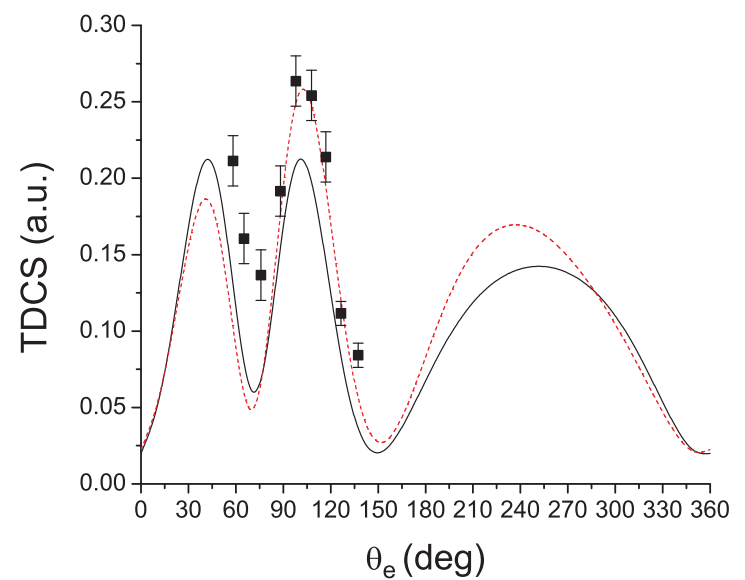

(a)

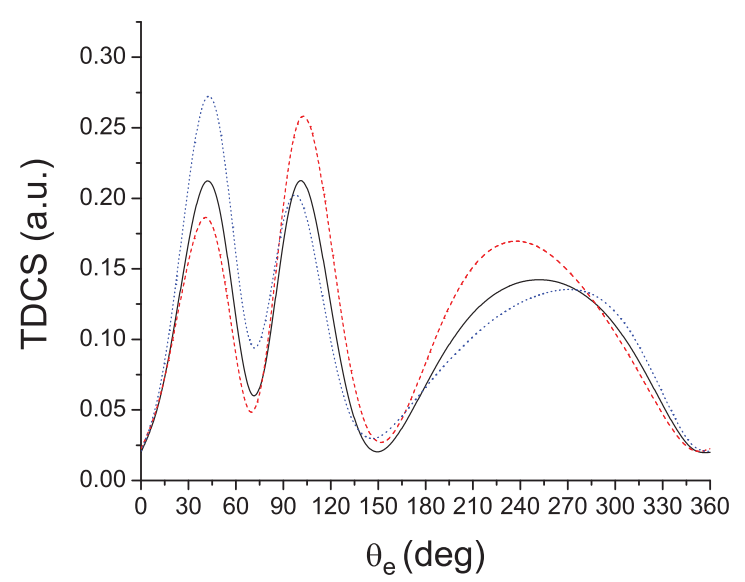

(b)

FIG. 4. (Color online) (a) Triple differential cross section for $250 \mathrm{eV}$ electron-impact ionization of the $1 b_{1}$ orbital of the water molecule. The energy of the ejected electron is $E_{e}=10 \mathrm{eV}$ and the scattered angle is $15^{\circ}$. The theoretical calculations are performed in the first Born approximation (solid line) and in the second Born approximation (dashed line). The experimental data are those of Milne-Brownlie et al. [3]. (b) Triple differential cross section for $250 \mathrm{eV}$ electron-impact and positron-impact ionization of the $1 b_{1}$ orbital of the water molecule. The energy of the ejected electron is $E_{e}=10 \mathrm{eV}$ and the scattered angle is $15^{\circ}$. The theoretical calculations are performed in the first Born approximation (solid line) and in the second Born approximation for electron impact (dashed line) and for positron impact (dotted line).

for electron and positron impact. Here we also observe that the first peak of the binary peak increases while the second peak decreases for the positron impact.

Figure 5(a) shows the results for the ionization of the $1 b_{2}$ state by electron impact. The SBA results are in better agreement with the experimental data of Milne-Brownlie et al. [3] than the FBA. Figure 5(b) for the electron and positron impact shows the same results as in Figs. 3(b) and 4(b): The symmetrical double-peak structure of the binary peak given by the first Born approximation is destroyed when the second Born approximation is applied. This result was also obtained with the BBK and DS3C models [17]. As a matter of fact, the three states $\left(3 a_{1}, 1 b_{1}\right.$, and $\left.1 b_{2}\right)$ are mainly built by the $L=1$ partial-wave expansion of the initial state of the wave

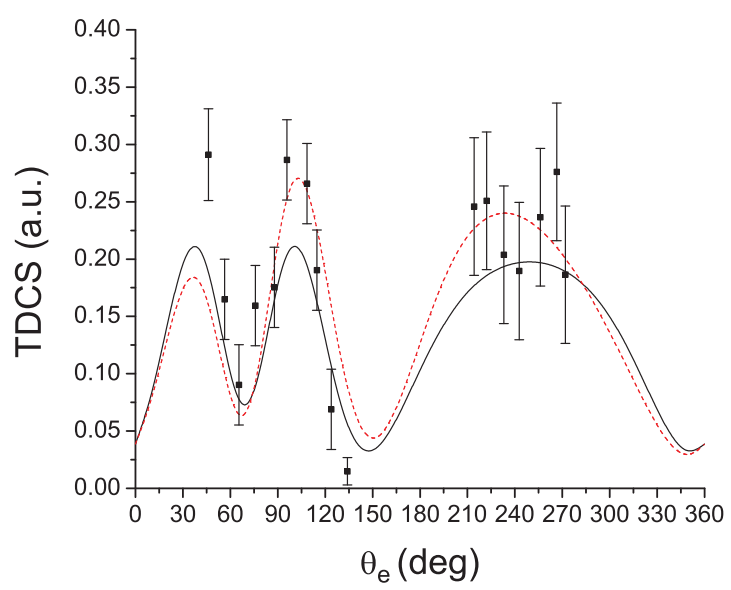

(a)

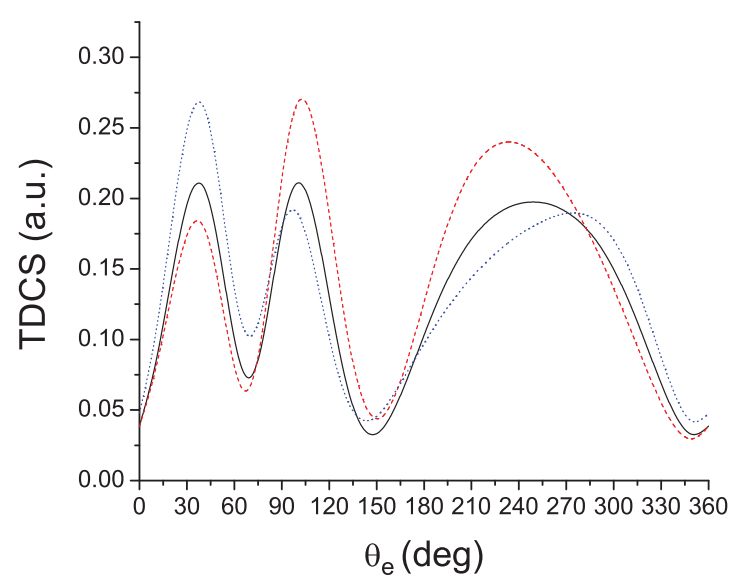

(b)

FIG. 5. (Color online) (a) Triple differential cross section for $250 \mathrm{eV}$ electron-impact ionization of the $1 b_{2}$ orbital of the water molecule. The energy of the ejected electron is $E_{e}=10 \mathrm{eV}$ and the scattered angle is $15^{\circ}$. The theoretical calculations are performed in the first Born approximation (solid line) and in the second Born approximation (dashed line). The experimental data are those of Milne-Brownlie et al. [3]. (b) Triple differential cross section for $250 \mathrm{eV}$ electron-impact and positron-impact ionization of the $1 b_{2}$ orbital of the water. The energy of the ejected electron is $E_{e}=10 \mathrm{eV}$ and the scattered angle is $15^{\circ}$. The theoretical calculations are performed in the first Born approximation (solid line) and in the second Born approximation for electron impact (dashed line) and for positron impact (dotted line).

function. We also observe that our second Born model with the closure approximation practically reproduces the same results as those given by the BBK or DS3C models. The agreement between these models and the experimental data [3] is very good. This good agreement is found in the vicinity of the Bethe ridge [36]. In this case the momentum transfer is close to the value of the momentum of the ejected electron $K \approx k_{e}$.

We now investigate the ionization of the thymine molecule [6]. Thymine has 66 electrons but our model can only be applied for the 48 electrons of the valence shells. For the remaining 18 electrons of the inner shells the convergence of our single-center expansion compared to the multicenter wave function is very slow. In this paper we investigate the 


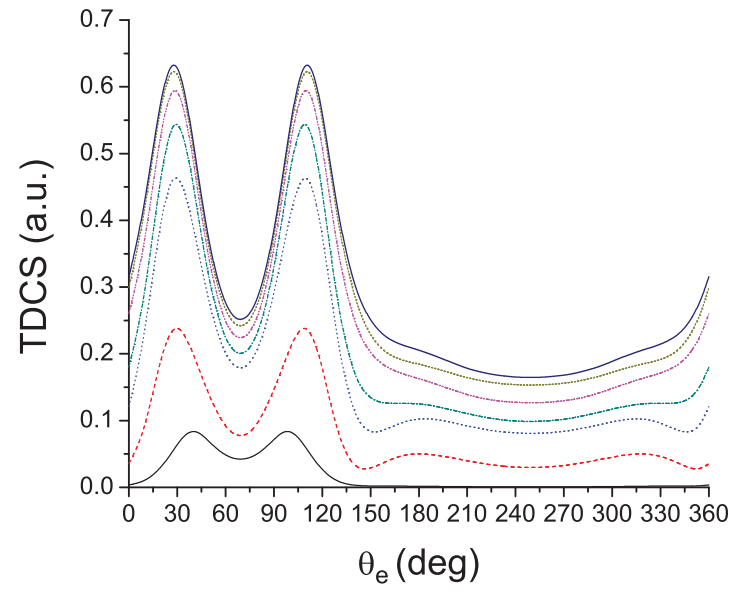

(a)

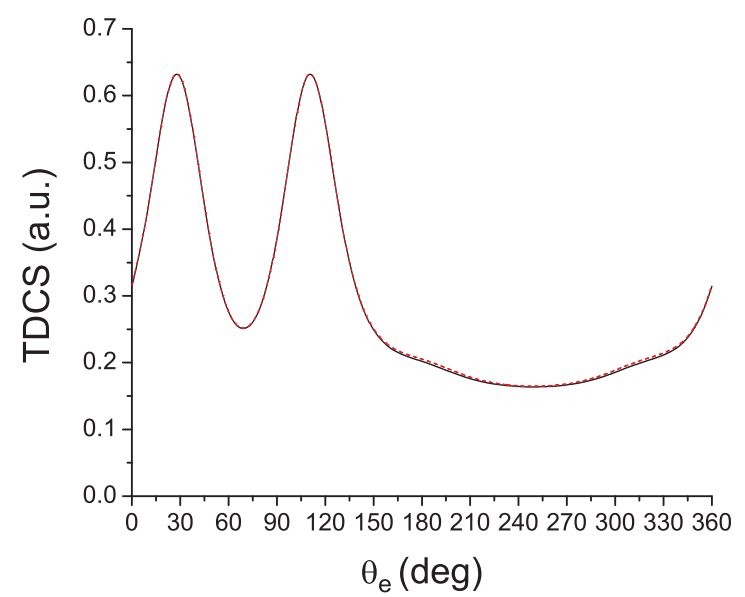

(b)

FIG. 6. (Color online) (a) Triple differential cross section for $250 \mathrm{eV}$ electron-impact ionization of the first valence orbital of the thymine (ionization energy: $I_{i}=9.14 \mathrm{eV}$ ). The energy of the ejected electron is $E_{e}=20 \mathrm{eV}$ and the scattered angle is $15^{\circ}$. The theoretical calculations are performed in the first Born approximation: partial wave $L=1$ (thin solid line), partial waves $L=1$ to $L=2$ (dashed line), partial waves $L=1$ to $L=3$ (dotted line), partial waves $L=1$ to $L=4$ (dashed-dotted line), partial waves $L=1$ to $L=5$ (dash-dot dotted line), partial waves $L=1$ to $L=6$ (short dashed line), and partial waves $L=1$ to $L=10$ (thick solid line). (b) Triple differential cross section for $250 \mathrm{eV}$ electron-impact ionization of the first valence orbital of the thymine (ionization energy: $I_{i}=9.14 \mathrm{eV}$ ). The energy of the ejected electron is $E_{e}=20 \mathrm{eV}$ and the scattered angle is $15^{\circ}$. The theoretical calculations are performed in the first Born approximation: partial waves $L=1$ to $L=8$ (thick solid line) and partial waves $L=1$ to $L=10$ (dashed line).

ionization of the last four shells for which the ionization potentials are estimated to be $9.14 \mathrm{eV}, 11 \mathrm{eV}, 11.4 \mathrm{eV}$, and $12.16 \mathrm{eV}$, respectively [7]. The experimental conditions are the following: The incident energy is $E_{i}=250 \mathrm{eV}$ and the energy of the ejected electron is $E_{e}=20 \mathrm{eV}$ while the geometrical conditions are given by $\phi_{s}=180^{\circ}, \phi_{e}=0^{\circ}$, and $\theta_{s}=15^{\circ}$ or $\theta_{s}=10^{\circ}$. The choice of $\theta_{s}=15^{\circ}$ practically corresponds to the Bethe ridge.

Next we check the convergence of our partial-wave expansion of the multicenter wave function by applying the first Born approximation for $\theta_{s}=15^{\circ}$ and for the last valence shell

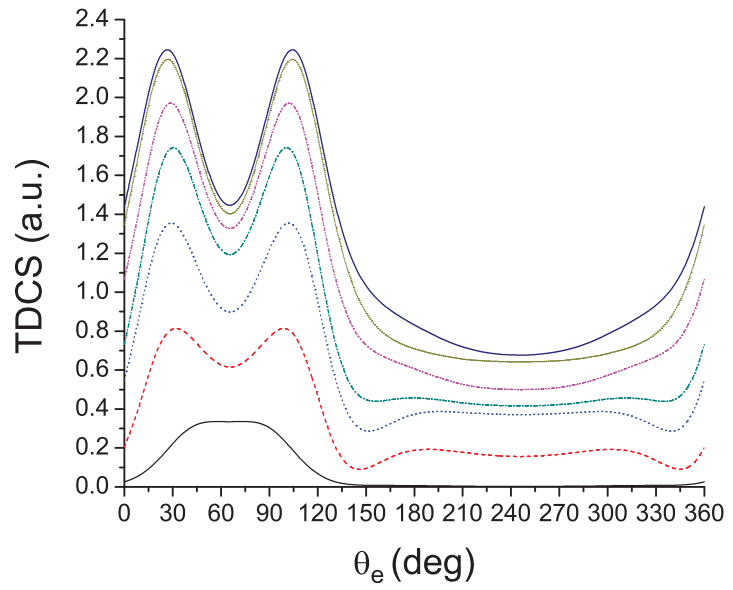

(a)

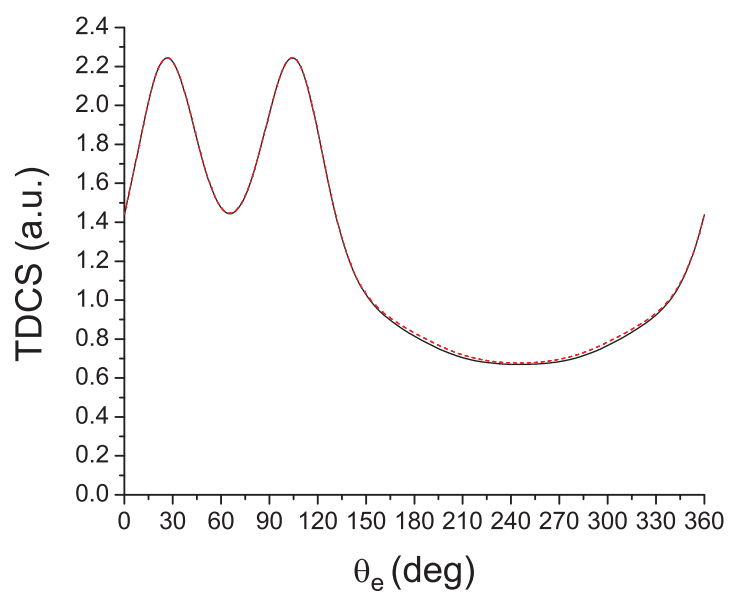

(b)

FIG. 7. (Color online) (a) Triple differential cross section for $250 \mathrm{eV}$ electron-impact ionization of the first valence orbital of the thymine (ionization energy: $I_{i}=9.14 \mathrm{eV}$ ). The energy of the ejected electron is $E_{e}=20 \mathrm{eV}$ and the scattered angle is $10^{\circ}$. The theoretical calculations are performed in the first Born approximation: partial wave $L=1$ (thin solid line), partial waves $L=1$ to $L=2$ (dashed line), partial waves $L=1$ to $L=3$ (dotted line), partial waves $L=1$ to $L=4$ (dash and dotted line), partial waves $L=1$ to $L=5$ (dash-dot dotted line), partial waves $L=1$ to $L=6$ (short dashed line), and partial waves $L=1$ to $L=10$ (thick solid line). (b) Triple differential cross section for $250 \mathrm{eV}$ electron-impact ionization of the first valence orbital of the thymine (ionization energy: $I_{i}=9.14 \mathrm{eV}$ ). The energy of the ejected electron is $E_{e}=20 \mathrm{eV}$ and the scattered angle is $10^{\circ}$. The theoretical calculations are performed in the first Born approximation: partial waves $L=1$ to $L=8$ (thick solid line) and partial waves $L=1$ to $L=10$ (dashed line).

$\left(I_{i}=9.14 \mathrm{eV}\right)$. Figures 6(a) and 6(b) clearly show that the convergence is reached at $L=8$ (from $L=1$ to $L=8$ ). In Fig. 6 we see a double-peak structure for the binary peak as in the cases of the ionization of the water molecule $\left(3 a_{1}, 1 b_{1}\right.$, and $1 b_{2}$ states). When we change the scattering angle $\left(\theta_{s}=10^{\circ}\right)$, Figs. 7(a) and 7(b) give the same curves as those in the previous case $\left(\theta_{5}=15^{\circ}\right)$ except the magnitude, which increases here. The convergence of our partial-wave expansion is reached at $L=8$ too. Another interesting fact is the small magnitude of the recoil peak contrary to the case of the ionization of water. It is certainly due to the higher value of the energy of the 


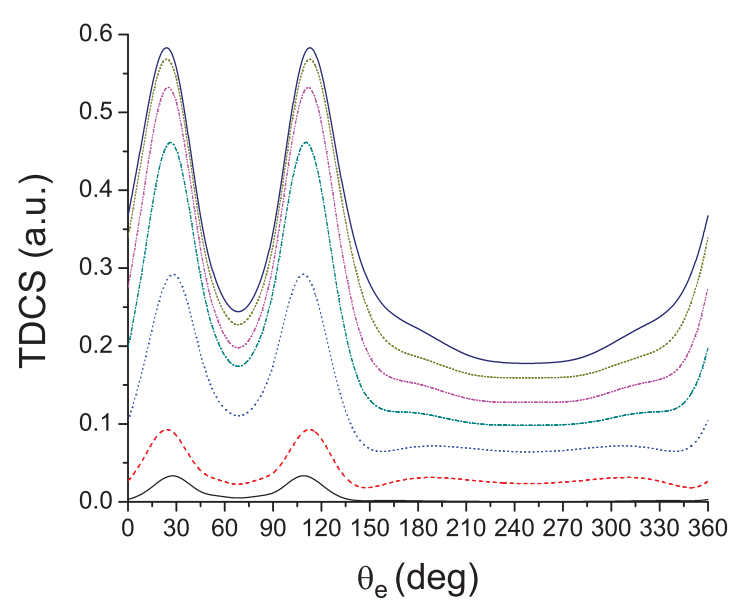

(a)

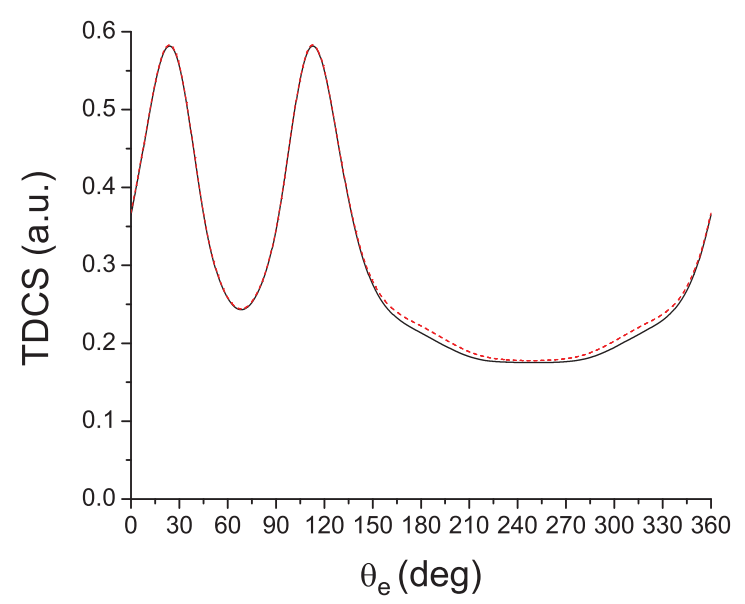

(b)

FIG. 8. (Color online) (a) Triple differential cross section for $250 \mathrm{eV}$ electron-impact ionization of the second valence orbital of the thymine (ionization energy: $I_{i}=11 \mathrm{eV}$ ). The energy of the ejected electron is $E_{e}=20 \mathrm{eV}$ and the scattered angle is $15^{\circ}$. The theoretical calculations are performed in the first Born approximation: partial wave $L=1$ (thin solid line), partial waves $L=1$ to $L=2$ (dashed line), partial waves $L=1$ to $L=3$ (dotted line), partial waves $L=1$ to $L=4$ (dashed- dotted line), partial waves $L=1$ to $L=5$ (dash-dot dotted line), partial waves $L=1$ to $L=6$ (short dashed line), and partial waves $L=1$ to $L=10$ (thick solid line). (b) Triple differential cross section for $250 \mathrm{eV}$ electron-impact ionization of the second valence orbital of the thymine (ionization energy: $I_{i}=11 \mathrm{eV}$ ). The energy of the ejected electron is $E_{e}=20 \mathrm{eV}$ and the scattered angle is $15^{\circ}$. The theoretical calculations are performed in the first Born approximation: partial waves $L=1$ to $L=8$ (thick solid line) and partial waves $L=1$ to $L=10$ (dashed line).

ejected electron here $(20 \mathrm{eV}$ instead of $8 \mathrm{eV}$ or $10 \mathrm{eV})$ because, generally speaking, the magnitude of the recoil peak increases when the energy of the ejected electron decreases. The results are very similar in the case of the ionization of the second outer shell $\left(I_{i}=11 \mathrm{eV}\right)$. The convergence is again reached at $L=8$ in the two cases $\left(\theta_{s}=15^{\circ}\right.$ or $\left.\theta_{s}=10^{\circ}\right)$ and the magnitudes are very close (Figs. 8 and 9). More interesting is the case of the third outer shell $\left(I_{i}=11.4 \mathrm{eV}\right)$. We observe a complete change in the shapes of the curves when we consider $\theta_{s}=15^{\circ}$ [Figs. 10(a) and 10(b)] and $\theta_{5}=10^{\circ}$ [Figs. 11(a) and 11(b)].

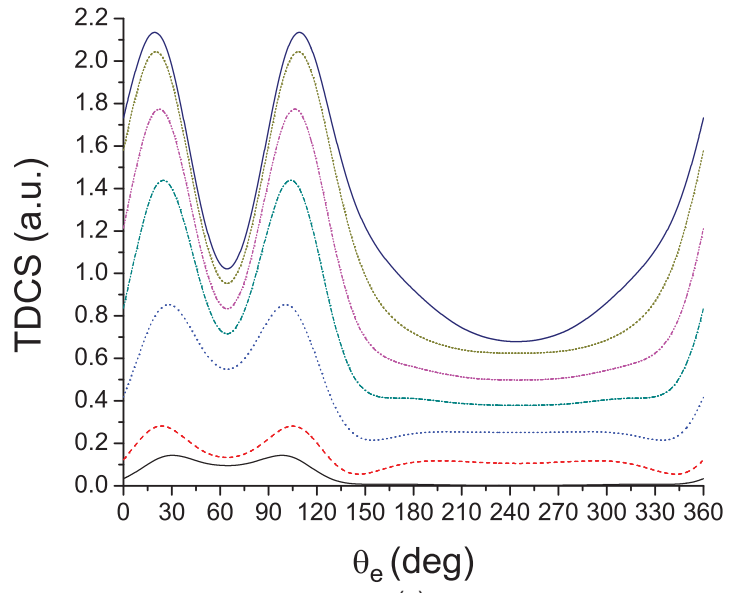

(a)

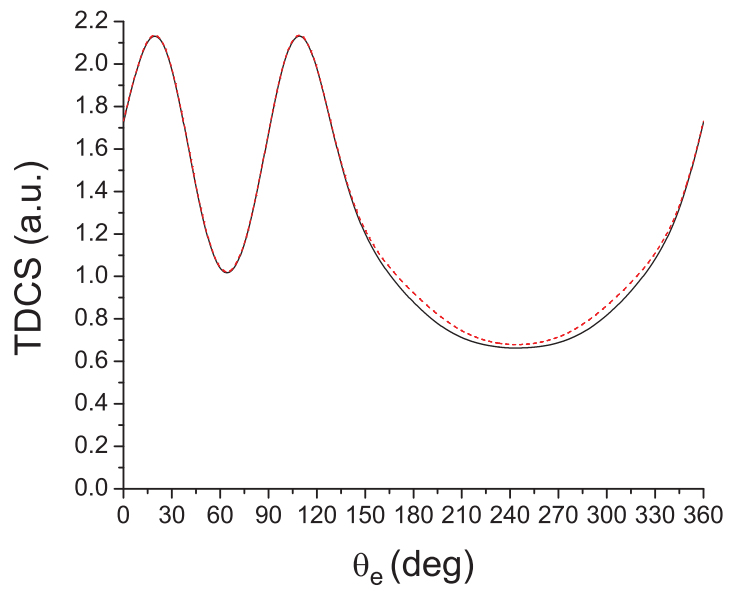

(b)

FIG. 9. (Color online) (a) Triple differential cross section for $250 \mathrm{eV}$ electron-impact ionization of the second valence orbital of the thymine (ionization energy: $I_{i}=11 \mathrm{eV}$ ). The energy of the ejected electron is $E_{e}=20 \mathrm{eV}$ and the scattered angle is $10^{\circ}$. The theoretical calculations are performed in the first Born approximation: partial wave $L=1$ (thin solid line), partial waves $L=1$ to $L=2$ (dashed line), partial waves $L=1$ to $L=3$ (dotted line), partial waves $L=1$ to $L=4$ (dash and dotted line), partial waves $L=1$ to $L=5$ (dash-dot dotted line), partial waves $L=1$ to $L=6$ (short dashed line), and partial waves $L=1$ to $L=10$ (thick solid line). (b) Triple differential cross section for $250 \mathrm{eV}$ electron-impact ionization of the second valence orbital of the thymine (ionization energy: $I_{i}=11 \mathrm{eV}$ ). The energy of the ejected electron is $E_{e}=20 \mathrm{eV}$ and the scattered angle is $10^{\circ}$. The theoretical calculations are performed in the first Born approximation: partial waves $L=1$ to $L=8$ (thick solid line) and partial waves $L=1$ to $L=10$ (dashed line).

In the first case $\left(\theta_{s}=15^{\circ}\right)$ we get a minimum in the direction of the momentum transfer (binary peak) while we get a plateau in the second case $\theta_{s}=10^{\circ}$. The convergence is reached for $L=9$ (from $L=0$ to $L=9$ ). More exciting are the results for the ionization of the fourth outer shell $\left(I_{i}=12.16 \mathrm{eV}\right)$. A rich complex structure is observed for the binary peak (four peaks) in Fig. $12\left(\theta_{s}=15^{\circ}\right)$ while we have only a single peak for $\theta_{s}=10^{\circ}$ (Fig. 13). The convergence is reached at $L=8$ for the binary peak but the recoil peak needs more terms (from $L=0$ to $L=15$ ). 


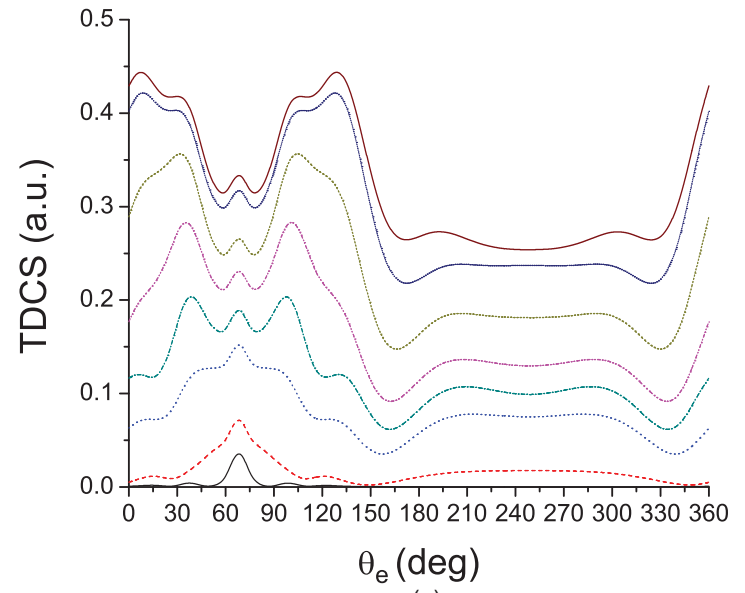

(a)

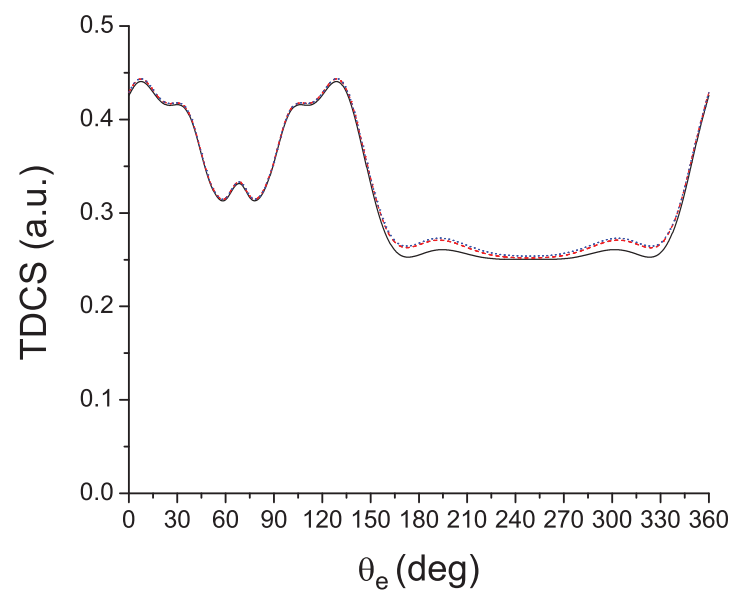

(b)

FIG. 10. (Color online) (a) Triple differential cross section for $250 \mathrm{eV}$ electron-impact ionization of the third valence orbital of the thymine (ionization energy: $I_{i}=11.4 \mathrm{eV}$ ). The energy of the ejected electron is $E_{e}=20 \mathrm{eV}$ and the scattered angle is $15^{\circ}$. The theoretical calculations are performed in the first Born approximation: partial wave $L=0$ (thin solid line), partial waves $L=0$ to $L=1$ (dashed line), partial waves $L=0$ to $L=2$ (dotted line), partial waves $L=0$ to $L=3$ (dashed-dotted line), partial waves $L=0$ to $L=4$ (dash-dot dotted line), partial waves $L=0$ to $L=5$ (short dashed line), partial waves $L=0$ to $L=6$ (short dotted line), and partial waves $L=0$ to $L=10$ (thick solid line). (b) Triple differential cross section for $250 \mathrm{eV}$ electron-impact ionization of the third valence orbital of the thymine (ionization energy: $I_{i}=11.4 \mathrm{eV}$ ). The energy of the ejected electron is $E_{e}=20 \mathrm{eV}$ and the scattered angle is $15^{\circ}$. The theoretical calculations are performed in the first Born approximation: partial waves $L=0$ to $L=8$ (solid line), partial waves $L=0$ to $L=9$ (dashed line), and partial waves $L=0$ to $L=10$ (dotted line).

From the above analysis it seems very interesting to study the third and fourth outer shells, which exhibit very complex structures for the binary peak in the Bethe ridge $\left(\theta_{s}=15^{\circ}\right)$. This complex structure disappears for $\theta_{s}=10^{\circ}$.

The second Born approximation requires a large amount of computer time. Typically the second Born approximation for the ionization of the water molecule needs six hours for one point while for the thymine molecule it is around 40 days. So we present only one figure for the ionization of the

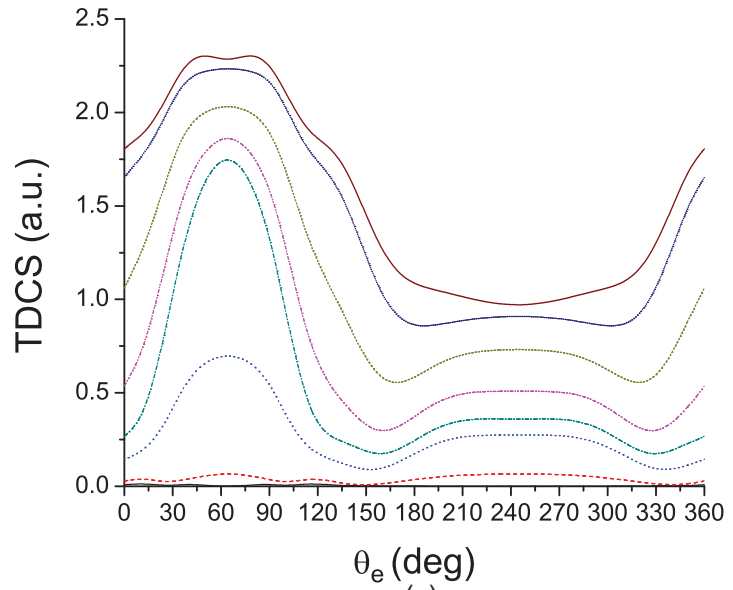

(a)

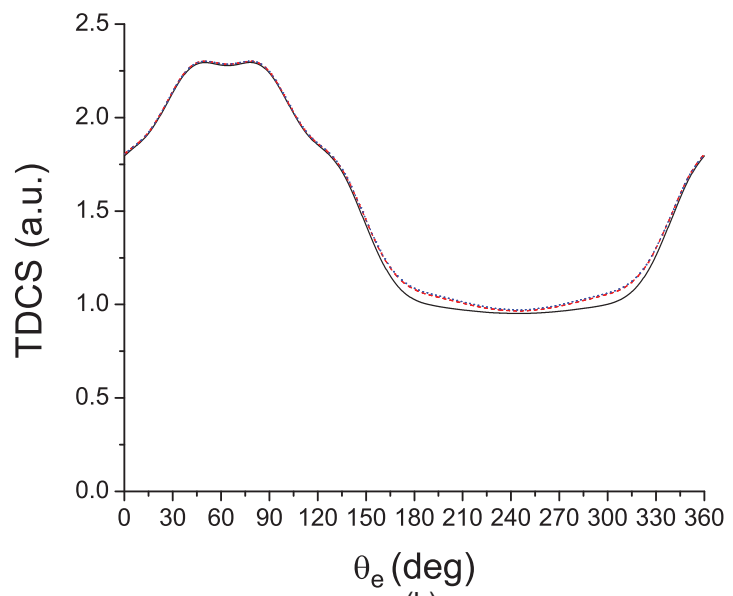

(b)

FIG. 11. (Color online) (a) Triple differential cross section for $250 \mathrm{eV}$ electron-impact ionization of the third valence orbital of the thymine (ionization energy: $I_{i}=11.4 \mathrm{eV}$ ). The energy of the ejected electron is $E_{e}=20 \mathrm{eV}$ and the scattered angle is $10^{\circ}$. The theoretical calculations are performed in the first Born approximation: partial wave $L=0$ (thin solid line), partial waves $L=0$ to $L=1$ (dashed line), partial waves $L=0$ to $L=2$ (dotted line), partial waves $L=0$ to $L=3$ (dashed-dotted line), partial waves $L=0$ to $L=4$ (dash-dot dotted line), partial waves $L=0$ to $L=5$ (short dashed line), partial waves $L=0$ to $L=6$ (short dotted line), and partial waves $L=0$ to $L=10$ (thick solid line). (b) Triple differential cross section for $250 \mathrm{eV}$ electron-impact ionization of the third valence orbital of the thymine (ionization energy: $I_{i}=11.4 \mathrm{eV}$ ). The energy of the ejected electron is $E_{e}=20 \mathrm{eV}$ and the scattered angle is $10^{\circ}$. The theoretical calculations are performed in the first Born approximation: partial waves $L=0$ to $L=8$ (solid line), partial waves $L=0$ to $L=9$ (dashed line), and partial waves $L=0$ to $L=10$ (dotted line).

first valence orbital of the thymine by electron and positron impacts. In Fig. 14 we notice that the symmetry around the momentum transfer is destroyed and that a double lobe of the binary peak appears. As in the case of the ionization of water the magnitude of the second peak increases for the electron impact. When we consider the ionization by positrons we see a change in the structure of the binary peak: The first peak increases. 


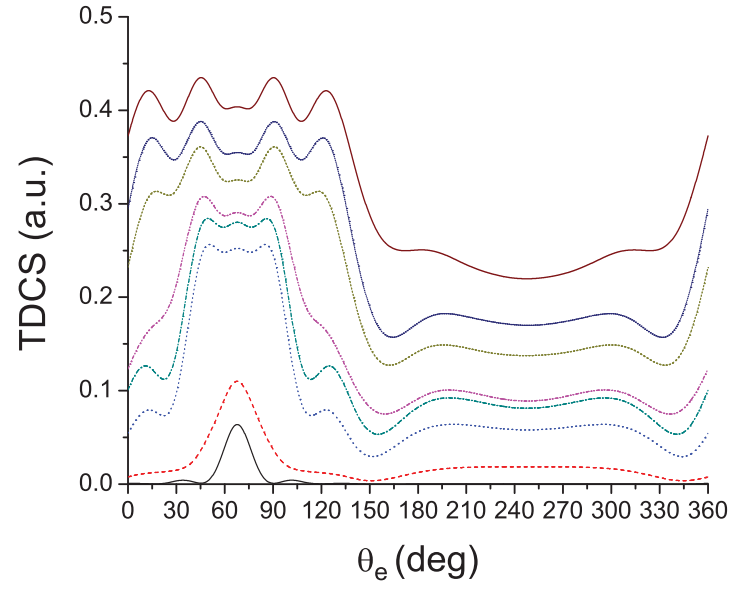

(a)

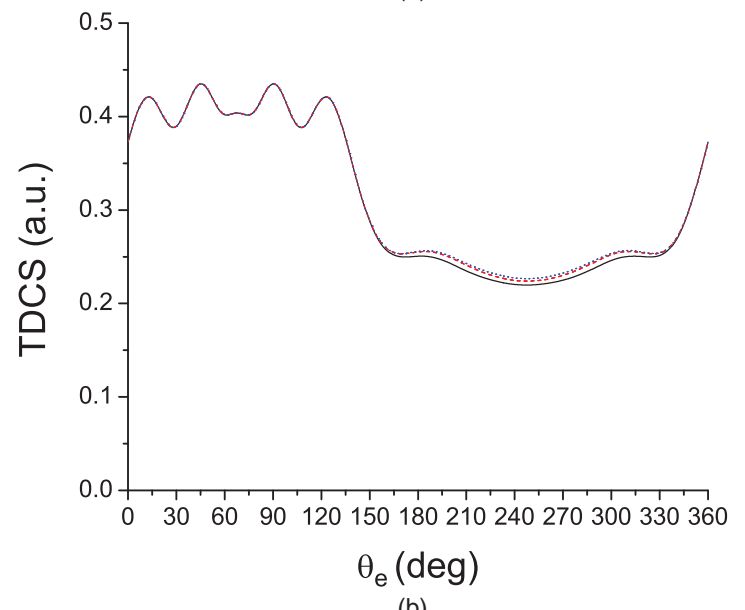

(b)

FIG. 12. (Color online) (a) Triple differential cross section for $250 \mathrm{eV}$ electron-impact ionization of the fourth valence orbital of the thymine (ionization energy: $I_{i}=12.16 \mathrm{eV}$ ). The energy of the ejected electron is $E_{e}=20 \mathrm{eV}$ and the scattered angle is $15^{\circ}$. The theoretical calculations are performed in the first Born approximation: partial wave $L=0$ (thin solid line), partial waves $L=0$ to $L=1$ (dashed line), partial waves $L=0$ to $L=2$ (dotted line), partial waves $L=0$ to $L=3$ (dashed-dotted line), partial waves $L=0$ to $L=4$ (dash-dot dotted line), partial waves $L=0$ to $L=5$ (short dashed line), partial waves $L=0$ to $L=6$ (short dotted line), and partial waves $L=0$ to $L=10$ (thick solid line). (b) Triple differential cross section for $250 \mathrm{eV}$ electron-impact ionization of the fourth valence orbital of the thymine (ionization energy: $I_{i}=12.16 \mathrm{eV}$ ). The energy of the ejected electron is $E_{e}=20 \mathrm{eV}$ and the scattered angle is $15^{\circ}$. The theoretical calculations are performed in the first Born approximation: partial waves $L=0$ to $L=10$ (solid line), partial waves $L=0$ to $L=12$ (dashed line), and partial waves $L=0$ to $L=30$ (dotted line).

\section{CONCLUSION}

Triple differential cross sections for the ionization of water and thymine molecules obtained in the second Born and eikonal approximation methods are presented and discussed. Our models use single-center wave functions and provide results in good agreement with other models (BBK and DS3C) and with the experimental data of Milne-Brownlie et al. [3] for the ionization of the water molecule. In this case the convergence of our partial-wave expansion of the initial multicenter wave function is very fast and limited to one term

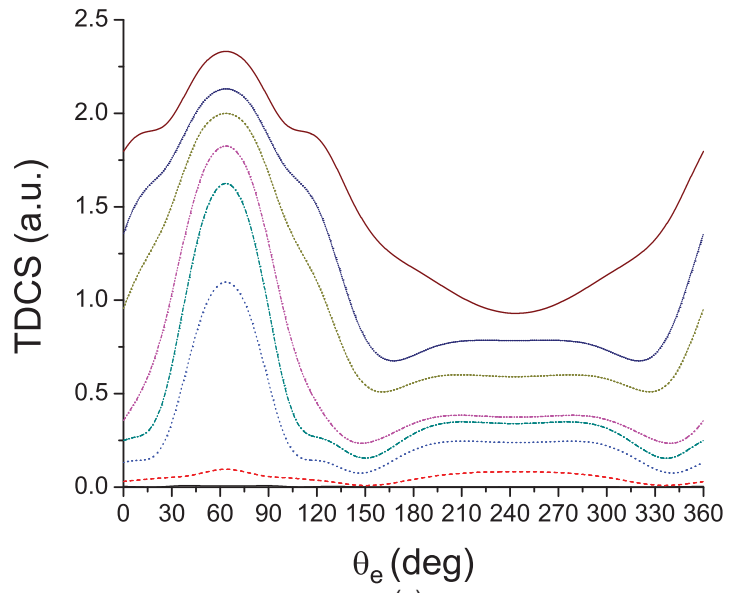

(a)

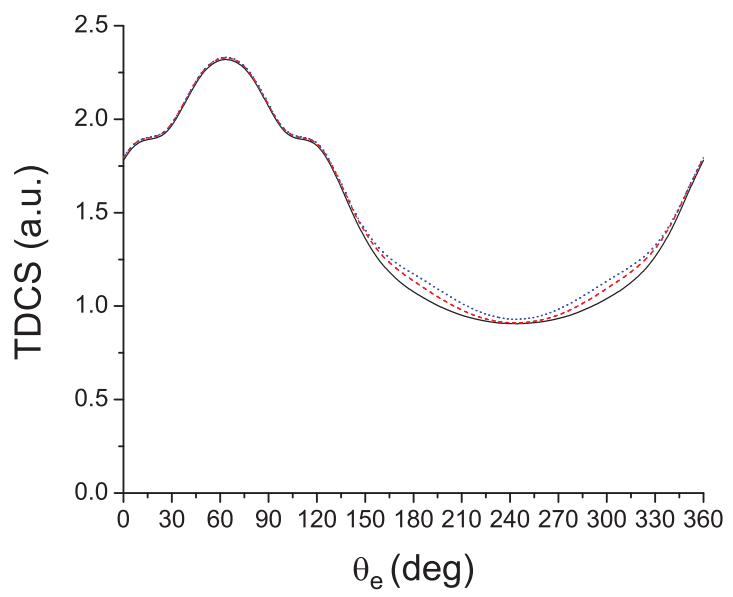

(b)

FIG. 13. (Color online) (a) Triple differential cross section for $250 \mathrm{eV}$ electron-impact ionization of the fourth valence orbital of the thymine (ionization energy: $I_{i}=12.16 \mathrm{eV}$ ). The energy of the ejected electron is $E_{e}=20 \mathrm{eV}$ and the scattered angle is $10^{\circ}$. The theoretical calculations are performed in the first Born approximation: partial wave $L=0$ (thin solid line), partial waves $L=0$ to $L=1$ (dashed line), partial waves $L=0$ to $L=2$ (dotted line), partial waves $L=0$ to $L=3$ (dashed-dotted line), partial waves $L=0$ to $L=4$ (dash-dot dotted line), partial waves $L=0$ to $L=5$ (short dashed line), partial waves $L=0$ to $L=6$ (short dotted line), and partial waves $L=0$ to $L=10$ (thick solid line). (b) Triple differential cross section for $250 \mathrm{eV}$ electron-impact ionization of the fourth valence orbital of the thymine (ionization energy: $I_{i}=12.16 \mathrm{eV}$ ). The energy of the ejected electron is $E_{e}=20 \mathrm{eV}$ and the scattered angle is $10^{\circ}$. The theoretical calculations are performed in the first Born approximation: partial waves $L=0$ to $L=8$ (solid line), partial waves $L=0$ to $L=9$ (dashed line), and partial waves $L=0$ to $L=10$ (dotted line).

( $L=0$ or $L=1$ ). In the case of thymine the ionization of the four last outer shells needs more terms in the expansion of the initial wave function (generally up to $L=8$ or $L=9$ ). We observe in some cases a rich complex structure in the curves in the Bethe ridge. All these results can now be an impetus for future experiments about the ionization of the thymine molecule by electrons. We also study the ionization of water and thymine molecules by positrons. When we compare the present cross sections with the results given by the first Born 


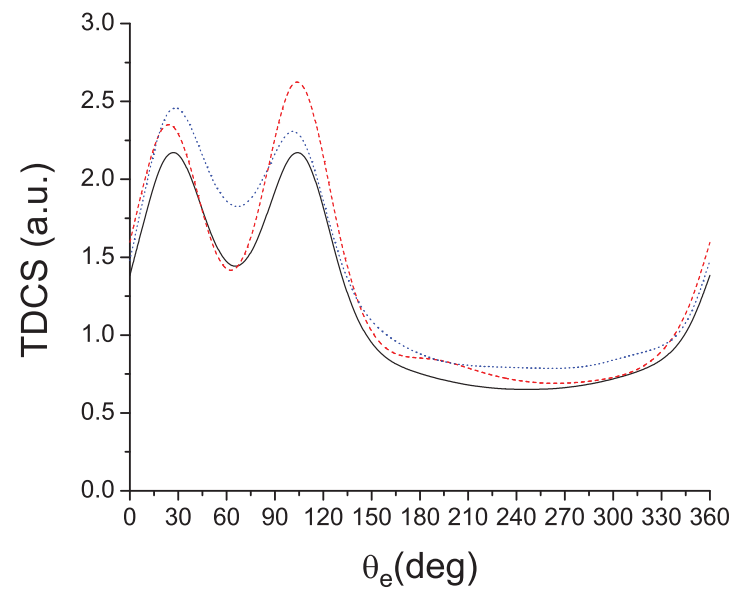

FIG. 14. (Color online) Triple differential cross section for $250 \mathrm{eV}$ electron-impact and positron-impact ionization of the first valence orbital of the thymine (ionization energy: $I_{i}=9.14 \mathrm{eV}$ ). The energy of the ejected electron is $E_{e}=20 \mathrm{eV}$ and the scattered angle is $10^{\circ}$. The theoretical calculations are performed in the first Born approximation (solid line) and in the second Born approximation for electron impact (dashed line) and for positron impact (dotted line).

approximation it is observed that the magnitude of the recoil peak decreases while that of the binary peak increases.

We hope that this work opens the way to experiments, especially for triple differential cross sections, which give the most accurate information about the mechanism of the ionization of an atom or a molecule. A knowledge of this mechanism is essential for the study of the penetration of charged particles through biological matter.

\section{ACKNOWLEDGMENT}

We would like to thank the Pôle Messin de Modélisation et de Simulation (PMMS) for computer time.

\section{APPENDIX}

In ab initio calculations of molecular electronic structure, the functions which are almost universally used by quantum chemists are the so-called contracted Gaussian-type orbitals (cGTOs), more specifically contracted Cartesian Gaussiantype orbitals (cCGTOs) of the form

$$
\varphi_{i}=\sum_{j=1}^{n_{p}} d_{i j} \chi_{j}
$$

where the coefficients $d_{i j}$ are the contraction coefficients and the functions $\chi_{j}$ are the primitive Cartesian Gaussian-type orbitals (CGTOs) called also Cartesian Gaussian. Generally the number of CGTOs varies between 1 and 7 (i.e., $1 \leqslant n_{p} \leqslant$ 7). These functions are defined as

$$
\begin{aligned}
\chi_{a}(x, y, z)= & N_{\alpha, i, j, k}\left(x-x_{A}\right)^{i}\left(y-y_{A}\right)^{j}\left(z-z_{A}\right)^{k} \\
& \times \exp \left(-\alpha\left|\vec{r}-\vec{R}_{A}\right|^{2}\right),
\end{aligned}
$$

where $a$ denotes the parameter set $a=\left(\alpha, \vec{R}_{A}, i, j, k\right), \vec{r}=$ $(x, y, z), \quad \vec{R}_{A}=\left(x_{A}, y_{A}, z_{A}\right)$ and $N_{\alpha, i, j, k}$ is a normalization constant such as

$$
N_{\alpha, i, j, k}=\left(\frac{2 \alpha}{\pi}\right)^{3 / 4}\left(\frac{2^{2(i+j+k)} \alpha^{i+j+k}}{(2 i-1) ! !(2 j-1) ! !(2 k-1) ! !}\right)^{1 / 2} .
$$

In the above expression the following definition is used: $(2 n) ! !=2 \times 4 \times 6 \times \ldots \times(2 n),(2 n+1) ! !=1 \times 3 \times$ $5 \times \ldots \times(2 n+1)$ and $0 ! !=(-1) ! !=1 ! !=1$. The parameter $\alpha$ controls the width of the orbital (a large (small) value gives a tight (diffuse) function) and $i, j, k$, control the angular momentum, $l=i+j+k$. Generally, quantum chemistry calculations are restricted to $l \leqslant 4$ (i.e., $g$ orbitals). Here $\Phi_{i}$ denotes a molecular orbital (e.g., $1 b_{1}$ of the water molecule) and is defined by

$$
\Phi_{i}(x, y, z)=\sum_{k=1}^{n_{c}} a_{i k} \varphi_{k},
$$

where $n_{c}$ is the number of cCGTOs, which is of the order of few hundred. The coefficients $a_{i k}$ are obtained from the program GAUSSIAN 03.

A spherical Gaussian-type orbital (SGTO) is defined as

$$
\begin{aligned}
\phi_{b}(\vec{r})= & N_{\alpha, n, l}\left|\vec{r}-\vec{R}_{A}\right|^{2 n+l} Y_{l, m}\left(\Omega_{\vec{r}-\vec{R}_{A}}\right) \\
& \times \exp \left(-\alpha\left|\vec{r}-\vec{R}_{A}\right|^{2}\right),
\end{aligned}
$$

with $b=\left(\alpha, \vec{R}_{A}, n, l, m\right)$, and

$$
N_{\alpha, n, l}=\left(2 \frac{(2 \alpha)^{2 n+l+3 / 2}}{\mathrm{~T}(2 n+l+3 / 2)}\right)^{1 / 2}
$$

a normalization constant.

The functions $\chi_{a}$ defined in Eq. (A2) and $\phi_{b}$ defined in Eq. (A5) are linked together through the transformation

$$
\chi_{a}(x, y, z)=\sum_{n, l, m} A(i j k ; n l m) \phi_{b}(\vec{r}),
$$

where the summation is restricted by the condition $2 n+l=$ $i+j+k$.

Most of the computer programs in the field of atomic and molecular collisions use the partial-wave expansion techniques. This means that the molecular wave functions must be expanded around a common center as follows:

$$
\Phi_{i}(\vec{r})=\sum_{\lambda, m_{\lambda}} \tilde{R}_{\lambda, m_{\lambda}}^{i}(r) Y_{\lambda, m_{\lambda}}\left(\Omega_{\vec{r}}\right) .
$$

The molecular orbitals coming out from a quantum chemistry program (such as GAUSSIAN 03) are expressed in terms of linear combination of cCGTOs $\left[\varphi_{k}\right.$ in Eq. (A4)], which are themselves linear combination of CGTOs $\left[\chi_{j}\right.$ in Eq. (A1)]. As we have seen previously these latter functions can be expressed in terms of SGTOs $\left[\phi_{b}\right.$ in Eq. (A7)] therefore the expansion in Eq. (A8) can be obtained by performing a single-center expansion of the SGTOs. Following $[25,37]$ we have

$$
\phi_{b}(\vec{r})=\sum_{l^{\prime}=0}^{\tilde{l}_{\max }+l} \sum_{m^{\prime}=-l^{\prime}}^{l^{\prime}} R_{l^{\prime}, m^{\prime}}(r) Y_{l^{\prime}, m^{\prime}}\left(\Omega_{\vec{r}}\right),
$$


or more explicitly

$$
\phi_{b}(\vec{r})=4 \pi N_{\alpha, n, l} R_{A}^{2 n} \exp \left[-\alpha\left(r^{2}+R_{A}^{2}\right)\right] \sum_{l_{1}, l_{2}=0}^{l} \sum_{\tilde{l}=0}^{\tilde{l}_{\max }} \sum_{l^{\prime}, l^{\prime \prime}} \sum_{m^{\prime}, m^{\prime \prime}} C\left(l_{1}, l_{2}, \tilde{l}, l^{\prime}, m^{\prime}, l^{\prime \prime}, m^{\prime} l, m\right) r^{l_{1}} R_{A}^{l_{2}} \varsigma_{\tilde{l}}^{(2 n)}\left(\alpha, r, R_{A}\right) Y_{l^{\prime \prime}, m^{\prime \prime}}\left(\Omega_{\vec{R}_{A}}\right) Y_{l^{\prime}, m^{\prime}}\left(\Omega_{\vec{r}}\right)
$$

with

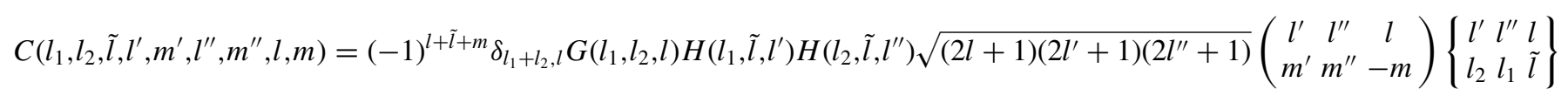

and

$$
G\left(l_{1}, l_{2}, l\right)=(-1)^{l_{2}}\left(\frac{4 \pi(2 l+1) !}{\left(2 l_{1}+1\right) !\left(2 l_{2}+1\right) !}\right)^{1 / 2}
$$

and

$$
H\left(l_{1}, \tilde{l}, l^{\prime}\right)=\left(\frac{\left(2 l_{1}+1\right)(2 \tilde{l}+1)}{4 \pi\left(2 l^{\prime}+1\right)}\right)^{1 / 2}\left\langle l_{1} \tilde{l} 00 \mid l^{\prime} 0\right\rangle
$$

where $\left\langle l_{1} \tilde{l} 00 \mid l^{\prime} 0\right\rangle$ is a Clebsch-Gordan coefficient.

Moreover

$$
\varsigma_{\tilde{l}}^{(2 n)}\left(\alpha, r, R_{A}\right)=4 \pi \sum_{l^{\prime \prime}=0}^{n} \sum_{l^{\prime}=\left|\tilde{l}-l^{\prime \prime}\right|}^{\tilde{l}+l^{\prime \prime}} H^{2}\left(l^{\prime}, l^{\prime \prime}, \tilde{l}\right) R_{l^{\prime \prime}}^{(2 n)}\left(r, R_{A}\right) i_{l^{\prime}}\left(2 \alpha r R_{A}\right)
$$

with

$$
R_{l^{\prime \prime}}^{(2 n)}\left(r, R_{A}\right)=\sum_{i=l^{\prime \prime}}^{2 n-l^{\prime \prime}} T_{l^{\prime \prime}, i}^{(2 n)}\left(\frac{r}{R_{A}}\right)^{i}
$$

and

$$
T_{l^{\prime \prime}, i}^{(2 n)}=(-1)^{l^{\prime \prime}} \frac{(2 n+1) !}{\left(i-l^{\prime \prime}\right) ! !\left(i+l^{\prime \prime}+1\right) ! !\left(2 n-i-l^{\prime \prime}\right) ! !\left(2 n-i+l^{\prime \prime}+1\right) ! !} .
$$

In the above equation the summation is performed in steps of 2 . In Eq. (A14) the functions $i_{l}(r)$ are the modified spherical Bessel functions [38] defined as

$$
i_{l}(r)=\sqrt{\frac{\pi}{2 r}} I_{l+1 / 2}(r)
$$

The presence of the $3 j$ and $6 j$ symbols in Eq. (A11) and the Clebsch-Gordan coefficient in Eq. (A13) leads to the following selection rules $\left|\tilde{l}-l_{1}\right| \leqslant l^{\prime} \leqslant \tilde{l}+l_{1},\left|\tilde{l}-l_{2}\right| \leqslant l^{\prime \prime} \leqslant \tilde{l}+l_{2},-l^{\prime} \leqslant m^{\prime} \leqslant l^{\prime}$, and $-l^{\prime \prime} \leqslant m^{\prime \prime} \leqslant l^{\prime \prime}$. The value of $\tilde{l}_{\text {max }}$ is chosen in order to ensure the convergence of Eq. (A10). Typically, $\tilde{l}_{\max } \approx 10$ is required for not too large molecules such as water.

[1] H. Hafied, A. Eschenbrenner, C. Champion, M.F. Ruiz-Lopez, C. Dal Cappello, I. Charpentier, and P. A. Hervieux, Chem. Phys. Lett. 439, 55 (2007).

[2] C. Dal Cappello, P. A. Hervieux, I. Charpentier, and F. RuizLopez, Phys. Rev. A 78, 042702 (2008).

[3] D. S. Milne-Brownlie, S. J. Cavanagh, B. Lohmann, C. Champion, P.-A. Hervieux, and J. Hanssen, Phys. Rev. A 69, 032701 (2004).

[4] C. Kaiser, D. Spieker, J. Gao, M. Hussey, A. J. Murray, and D. H. Madison, J. Phys. B 40, 2563 (2007).

[5] K. L. Nixon, A. J. Murray, O. Al-Hagan, D. H. Madison, and C. Ning, J. Phys. B 43, 035201 (2010).

[6] C. J. Colyer, S. M. Bellm, F. Blanco, G. Garcia, and B. Lohmann, J. Phys.: Conf. Ser. 288, 012014 (2011).
[7] Ph. Bernhardt and H. G. Paretzke, Int. J. Mass Spectrom. 223, 599 (2003).

[8] H. Deutsch, K. Becker, S. Matt, and T. D. Märk, Int. J. Mass Spectrom. 223, 599 (2003).

[9] Y. K. Kim, M. E. Rudd, Phys. Rev. A 50, 3954 (1994).

[10] I. Shafranyosh, M. I. Sukhoviya, and M. I. Shafranyosh, J. Phys. B 39, 4155 (2006).

[11] W. M. Huo, Phys. Rev. A 64, 042719 (2001).

[12] W. M. Huo, C. E. Dateo, and G. D. Fletcher, Radiat. Measurements 41, 1202 (2006).

[13] J. Gao, D. H. Madison, and J. L. Peacher, J. Chem. Phys. 123, 204314 (2005).

[14] D. H. Madison and O. Al-Hagan, J. At. Mol. Phys. 2010, 367180 (2010). 
[15] A. Prideaux and D. H. Madison, Phys. Rev. A 67, 052710 (2003).

[16] C. Champion, J. Hanssen, and P.-A. Hervieux, Phys. Rev. A 65, 022710 (2002).

[17] C. Champion, C. Dal Cappello, S. Houamer, and A. Mansouri, Phys. Rev. A 73, 012717 (2006).

[18] M. Brauner, J. S. Briggs, and H. Klar, J. Phys. B 22, 2265 (1989).

[19] V. Cobut, Y. Frongillo, J. P. Patau, T. Goulet, M. J. Frazer, and J. P. Jay-Gerin, Radiat. Phys. Chem. 51, 229 (1998).

[20] B. Boudaïffa, P. Cloutier, D. Hunting, M. A. Huels, and L. Sanche, Science 287, 1658 (2000).

[21] F. W. Byron Jr and C. J. Joachain Phys. Rev. 146, 1 (1966).

[22] R. J. Glauber, in Lectures in Theoretical Physics, edited by W. E. Brittin and L. G. Dunham (Interscience, New York, 1959), Vol. 1, p. 315.

[23] R. Dey and A. C. Roy, NIMB 243, 28 (2006), and references therein.

[24] M. J. Frisch et al., Gaussian 03, Revision B.05 (Gaussian, Inc., Wallingford, Connecticut, 2004).

[25] K. Kaufmann, W. Baumeister and M. Jungen, J. Phys. B 22, 2223 (1989).
[26] R. Moccia, J. Chem. Phys. 40, 2186 (1964).

[27] M. J. Brothers and R. A. Bonham, J. Phys. B 17, 4235 (1984).

[28] C. Dal Cappello, A. Haddadou, F. Menas, and A. C. Roy, J. Phys. B 44, 015204 (2011).

[29] C. Champion, J. Hanssen and P. A. Hervieux, Phys. Rev. A 63, 052720 (2001).

[30] C. Champion, J. Hanssen and P. A. Hervieux, J. Chem. Phys. 121, 9423 (2004).

[31] D. S. F. Crothers and J. F. McCann, J. Phys. B 16, 3229 (1983).

[32] S. Jones and D. H. Madison, Phys. Rev. Lett. 81, 2886 (1998).

[33] J. Berakdar and J. S. Briggs, Phys. Rev. Lett. 72, 3799 (1994).

[34] S. Zhang, J. Phys. B 33, 3545 (2000).

[35] H. Ray and A. C. Roy, Phys. Rev. A 46, 5714 (1992).

[36] A. Lahmam-Bennani, A. Naja, E. M. Staicu Casagrande, N. Okumus, C. Dal Cappello, I. Charpentier and S. Houamer, J. Phys. B 42, 165201 (2009).

[37] E. O. Steinborn and E. Filter, Theoret. Chim. Acta (Berl.) 38, 247 (1975).

[38] M. Abramowitz and I. A. Stegun, Handbook of Mathematical Functions (Dover, New York, 1972). 